\title{
Mapping lateral changes in conductance of a thin sheet using time-domain inductive electromagnetic data
}

\author{
Michal Kolaj ${ }^{1}$ and Richard Smith ${ }^{1}$
}

\begin{abstract}
With the inductive electromagnetic geophysical method, the laterally varying conductance of thin sheet models can be estimated either through a direct transform of the measured data or through inversion. The direct transform (called the simplified solution) does not require grid or line data and is simple enough to be performed in the field because the conductance at a location is calculated directly from the ratio of two measured magnetic fields (the vertical spatial and temporal derivative of the vertical magnetic field) at that location. However, the simplified solution assumes that the secondary horizontal magnetic fields are zero and/or that the sheet has a uniform conductance. Our nonapproximate solution (called the full inversion) does not make these assumptions, but requires gridded data, measurements of the secondary horizontal magnetic fields, and more complicated inversion algorithms. Through forward modeling, we found that the full inversion provides better results than the
\end{abstract}

simplified solution when the spatial gradient of the resistance is strong and/or when the horizontal magnetic fields are large. Because the simplified solution may be preferable due to its simplicity, we introduce two unreliability parameters, which assess the unreliability of the conductance calculated using the simplified solution. A comparison of the simplified solution and full inversion in a fixed in-loop survey collected overtop a dry tailings pond in Sudbury, Ontario, Canada, revealed that there were small differences around large conductance contrasts, which coincided with elevated unreliability parameters. The simplified solution is recommended if fast in-field interpretations are required, or additionally, as a first-pass survey that can be performed with sparse station spacing to identify areas of interest. Denser grid data can then be collected, for the more reliable full inversion, over areas of interest and/or zones where the simplified solution is expected to be unreliable as predicted by the unreliability parameters.

\section{INTRODUCTION}

The primary goal of most electromagnetic (EM) geophysical surveys is to determine the distribution of electrical properties, namely, conductivity, below the surface, which accurately explains the measured EM response (generally, the magnetic field or its time derivative). This is often accomplished through the use of stitched 1D conductivity-depth sections (either through 1D inversions and/or conductivity depth imaging/transforms; Macnae, 2007) and/or trial-and-error forward modeling whereby the interpreter adjusts synthetic models until the generated synthetic data resemble the field data (Hohmann and Raiche, 1987). Three-dimensional inversions such as those proposed by Haber et al. (2007) and/or Zhdanov (2010) are appealing because they more accurately describe the earth's geometry and the physics in more complex situations. However, these full 3D inversions have been hampered by the size, ill posedness, and complexity of the problem, and as such, the faster and simpler 1D algorithms are generally used (Macnae, 2007). In parallel, the thin-sheet approximation, which assumes that current is constrained to flow in a sheet that is inductively thin (Price, 1949), has been used extensively to simplify the equations used to solve for the EM fields in 3D media and has been effective in forward modeling and inversion routines (West et al., 1984; Macnae and Lamontagne, 1987; Keating and Crossley, 1990; Nabighian and Macnae, 1991; Liu and Asten, 1993; Smith, 2000; Tartaras et al., 2000; Swidinsky and Edwards, 2009; Kolaj and Smith, 2013). The success of the thin-sheet assumption is due to many factors, namely, the magnetic fields from a thin sheet are easy to visualize; many mineral exploration targets can be represented as thin sheets (Grant and West, 1965; Palacky,

\footnotetext{
Manuscript received by the Editor 12 June 2013; revised manuscript received 29 August 2013; published online 5 November 2013.

${ }^{1}$ Laurentian University, Department of Earth Sciences, Sudbury, Ontario, Canada. E-mail: mx_kolaj@laurentian.ca; rssmith@laurentian.ca.

(C) 2013 Society of Exploration Geophysicists. All rights reserved.
} 
1987); sedimentary layers can, as a first approximation, be thought of as stacked thin sheets (Tartaras et al., 2000; Walker and Lamontagne, 2007) and thin sheets are described by a conductance (product of conductivity and thickness), which is, at times, more robust than the corresponding conductivity and thickness (Liu and Asten, 1993). The thin-sheet approximation is relatively robust and generally valid as long as the thickness of the sheet is smaller than the skin depth or diffusion depth in the frequency and time domains, respectively (Joshi et al., 1988; Frischknecht et al., 1991). As such, while solving the full $3 \mathrm{D}$ inversion is sometimes preferred in terms of its accuracy, an inversion scheme based on thin sheets is an attractive shortcut in circumstances where the geology is consistent with the inherent assumptions.

A simple yet robust method for mapping a laterally varying conductance over extensive thin sheets from time-domain EM (TDEM) data was developed in Kolaj and Smith (2013) and successfully tested in a field trial over a dry mine tailings pond. Such an algorithm is useful and is a geologically reasonable approximation in many areas such as in nickel laterite exploration where the nickel content is at times associated with areas of high conductance in the variable saprolite (Peric, 1981; Rutherford et al., 2001); in the characterization of mine, mill, or smelter waste (Chouteau et al., 2006; Kolaj and Smith, 2013), and/or in the characterization or exploration over variable overburden (Seigel and Pitcher, 1978; Irvine and Staltari, 1984). The method of Kolaj and Smith (2013) involves a simplification of the EM induction formula for thin sheets derived by Price (1949) and generalized for EM prospecting methods by Smith and West (1987). The simplification assumed a uniform resistance (inverse of conductance) and calculated an apparent resistance in sheets where the resistance varied laterally. This assumption relied on the product of the horizontal magnetic fields with the corresponding derivatives of the lateral resistance (i.e., $H_{y}^{s} d R / d y$ and $H_{x}^{s} d R / d x$ ) being negligible in comparison to the other two terms in the original equation $\left(R d H_{z}^{s} / d z\right.$ and $\left.d H_{z} / d t\right)$. The advantages of the simplified over the nonsimplified method are (1) the horizontal magnetic fields $\left(H_{y}^{s}\right.$ and $H_{x}^{s}$ ) are not required, (2) the resistance calculated at each station is independent of any other station and thus line/grid data are not required, and (3) the resistance calculation is simple enough to be performed in the field in real time. However, the benefits of the simplified method are limited by the approximations made and thus a detailed study of when the simplified, or nonsimplified, solutions should be used is warranted.

We begin by briefly summarizing the solution to the problem of EM induction in thin sheets with a laterally varying conductance and how the equation may be inverted to estimate an apparent resistance (henceforth called the simplified solution) or the actual resistance (henceforth called the full inversion). Through forward modeling, we explore the general characteristics of each method and the limitations of the simplified solution and we present a simple calculation that can determine whether the resistance calculated with the simplified solution will be valid. Last, we use our developed full inversion and compare it to the simplified solution on the field data collected in Kolaj and Smith (2013) overtop a dry mine tailings pond situated on Vale property in Sudbury, Ontario, Canada.

\section{THEORY}

In Kolaj and Smith (2013), a simple method to estimate the apparent resistance of a horizontal inductively thin sheet embedded in a resistive medium is derived based on the work of Price (1949) and Smith and West (1987). The formula for the apparent resistance $R$ is

$$
R=\frac{\mu}{2}\left(\frac{\frac{d H_{z}}{d t}}{\frac{d H_{z}^{s}}{d z}}\right)
$$

where $\mu$ is the magnetic permeability (assumed to be that of free space) and $d H_{z} / d t$ and $d H_{z}^{s} / d z$ are the time derivative of the vertical total magnetic field (secondary plus primary magnetic field) and the vertical spatial derivative of the vertical secondary magnetic field, respectively. This apparent resistance estimate assumed that there was no change in resistance in the lateral directions (i.e., $d R / d y \approx 0$ and $d R / d x \approx 0$ ), which allowed simplification of the full thin-sheet induction boundary equation,

$$
-\frac{d H_{z}^{s}}{d z} R+\frac{d R}{d y} H_{y}^{s}+\frac{d R}{d x} H_{x}^{s}=-\frac{\mu}{2} \frac{d H_{z}}{d t} .
$$

In the off-time or when the primary magnetic field is a constant value, all terms in equations 1 and 2 are secondary magnetic fields $\left(\mathbf{H}^{S}\right)$ and the calculated resistance becomes independent of the transmitter location and waveform (Kolaj and Smith, 2013). However, when dealing with finite-sized and nonuniform conducting thin sheets, certain transmitter and receiver configurations will provide better results. For instance, the simplified solution (equation 1) will work best when $H_{y}^{s} d R / d y$ and $H_{x}^{s} d R / d x$ are small in comparison to $d H_{z} / d t$ and $d H_{z}^{s} / d z$, which is achievable when the magnetic fields are measured inside of the transmitter loop where the vertical magnetic fields dominate (Kolaj and Smith, 2013). Moreover, equations 1 and 2 are true at all delay times, so multiple resistance profiles, one for each time channel, can be calculated. The results from each time channel should be comparable unless the inductively thin sheet criteria (and uniform resistance for equation 1) assumptions are invalid. The results from each time channel can also differ if there are changes in resistivity with depth. While the simplified solution (equation 1) is valid away from the sheet, equation 2 is valid only in the plane bounded by the sheet (i.e., immediately above or below the conductive thin sheet). However, the magnetic fields can be calculated away from the sheet by upward continuing the fields calculated at the surface of the sheet using equation 2 (i.e., the forward model of Smith and West, 1987). Thus, using equation 2 to calculate the resistance using magnetic fields measured above the sheet (i.e., the inverse problem) should provide a smoothed approximate estimate of the true resistance. As such, for near-surface applications, no knowledge of the sheet depth is required to solve the simplified solution for the apparent resistance (equation 1) or to get an approximate resistance that satisfies equation 2. Furthermore, the methods will be limited to near-surface applications (where equation 2 is valid) as spatial derivatives (i.e., $d H_{z}^{s} / d z$ ) from deeper sources will likely be below background noise levels (Sattel and Macnae, 2001). Although equation 1 (the simplified solution) was used quite successfully in Kolaj and Smith (2013), the incorporation of the terms involving resistance derivatives (equation 2) should provide a better estimate of the true resistance where these terms cannot be neglected. 


\section{INVERSION SCHEME}

Equation 2 can be reformulated into a linear problem of the form

$$
\mathbf{A R}=\mathbf{b},
$$

where $\mathbf{R}$ is the vector of resistances at different locations on the sheet, $\mathbf{A}$ is a matrix corresponding to the left-hand side of equation 2 with finite-difference operators used to estimate the lateral resistance derivatives, and $\mathbf{b}$ is the vector of elements corresponding to the right-hand side of equation 2. The matrix $\mathbf{A}$ is sparse and can be inverted allowing for equation 3 to be solved, but, like many other geophysical problems, it is often ill conditioned and benefits from regularization. Thus, the inversion is performed by minimizing $f(\mathbf{R})$ in the overdetermined system

$$
f(\mathbf{R})=\|\mathbf{W}(\mathbf{A R}-\mathbf{b})\|^{2}+\alpha^{2}\|\mathbf{S R}\|^{2},
$$

where $\| .$. . $\mid$ represents the Euclidean norm, $\mathbf{W}$ is a weighting matrix corresponding to the inverse of the data error (assumed to be Gaussian distributed), $\mathbf{S}$ is a smoothing regularization matrix, and $\alpha$ is the regularization coefficient that controls the relative contribution between term 1 (misfit) and term 2 (smoothness) in equation 4 (a high value of $\alpha$ would produce a smoother model). Equation 4 is solved in MATLAB using orthogonal-triangular decomposition, and 1600 station resistances can be calculated in a few seconds on a desktop computer. Because the solution to equation 4 can be found quite rapidly, it can be rerun for many different values of $\alpha$. The optimum $\alpha$ is found using a combination of an L-curve analysis (Zhdanov, 2002) and a qualitative analysis of the solutions obtained. Note that equation 1 can be similarly reformulated into the form of equation 4 to benefit from the error weighting and regularization. In this case, the A matrix becomes a diagonal matrix. However, by reformulating the problem in this manner, the simplified solution would lose some of its advantages, namely, the independence of the calculated resistance from station to station, not requiring grid/line data and being able to calculate the resistance by taking a simple ratio of the measured fields.

The resistance derivatives (equation 2) are represented using a finite-difference approximation. Forward or backward differences are used along the edge of the grid, while a central difference is used within the grid. When there is a large range of values in the measured fields and derivatives and/or high noise in the data, erratic/erroneous resistances are sometimes calculated along the edge of the grid, which may propagate toward the center of the grid through the derivatives. An increase in the regularization coefficient tends to reduce this effect but may cause an overly smooth solution to be calculated. An alternative means of dealing with this issue is to estimate the measured fields, derivatives and the resistances beyond the survey area (we call this data padding). Data padding tends to move any potentially erratic resistances to be calculated in the padded zone, which is subsequently removed and thus, it is not interpreted. The simplest and most effective method is to duplicate the measured components $\left(d H_{z}^{s} / d z, H_{y}^{s}, H_{x}^{s}\right.$, and $\left.d H_{z} / d t\right)$ along the outer edge of the grid outward into the padded area. With the simplified solution, this would create a constant resistance envelope around the survey area.

\section{SYNTHETIC EXAMPLES}

In the following section, we discuss the accuracy and reliability of the estimates of resistance from the simplified solution (equations 1 and 4) and full inversion (equations 2 and 4) for three synthetic cases. As Kolaj and Smith (2013) suggest, the simplified solution is most effective when the survey is performed inside of the loop because in this configuration, the vertical magnetic fields will be large relative to the horizontal magnetic fields (i.e., the ignored terms $H_{y}^{s} d R / d y$ and $H_{x}^{s} d R / d x$ will be minimized). As such, the first example involves this configuration while the second and third examples involve loop positions outside of the survey area where the simplified solution may fail because the size of the ignored terms will be larger. In each example, a $30 \mathrm{~Hz}$, two-coil ground UTEM survey (with one receiver coil at $0 \mathrm{~m}$ and the second directly above at $2 \mathrm{~m}$ ) with 27 lines and 27 stations per line $(260 \mathrm{~m}$ line length, $10 \mathrm{~m}$ spacing between lines) was simulated in MultiLoop III (Lamontagne Geophysics; Walker and Lamontagne, 2006). See Figure 1 for a schematic of the transmitter-loop locations and the thin-sheet models used. Depths ranging from 20 to $40 \mathrm{~m}$ were used for the synthetic models because these near-surface sheets would be the typical target depths/thicknesses for this method (i.e., overburden, mine waste, and near-surface deposits). The vertical spatial derivative $\left(d H_{z}^{s} / d z\right)$ and the vertical-component time derivative $\left(d H_{z} / d t\right)$ were calculated using finite-difference operators. The identity matrix was used for the error matrix, and there was no noise added to the data (apart from some numerical noise present in the forward model).

\section{Example 1}

In the first example, a $400 \times 400 \mathrm{~m}$ loop is positioned around the survey area, the sheet is at a depth of $25 \mathrm{~m}$, and the background sheet and anomaly $(r=40 \mathrm{~m})$ are 0.5 and $0.05 \mathrm{ohm}$, respectively (Figure 1). The resistance results for the simplified solution and the full inversion gridded along the 27 lines can be seen in Figure 2. The regularization coefficient $\alpha$ was set to zero (i.e., the simplified solution is calculated directly for each station using equation 1), and no data padding was required.

At early time in particular, the background resistance of the sheet from the simplified solution and full inversion is estimated to be $0.5 \mathrm{ohm}$, which is consistent with the input value. The background resistance is underpredicted with increasing delay time with both methods (i.e., the background resistance in the later time channel is closer to $0.4 \mathrm{ohm}$ than the true value of $0.5 \mathrm{ohm}$ ), and it is hypothesized in Kolaj and Smith (2013) that it is due to the fields at a later delay time being impacted by the finite size of the sheet used in MultiLoop III. The dimensions of the anomaly are not as easy to estimate because they depend on which method and time channel are used. The simplified solution appears to produce sharper anomalies, but this is slightly deceiving because outside the anomalous area, the resistance overshoots the conductive anomaly (Figure 2). If the anomaly had been resistive, there would have been an undershoot (not shown). This overshoot and undershoot are most evident at late delay times. On the other hand, the full inversion provides smooth changes in the correct sense (i.e., broader anomalies with no over/underpredictions). Nevertheless, the anomaly size can be estimated to be roughly $80-120 \mathrm{~m}$ in diameter, which is consistent with the synthetic model. The resistance of the anomaly is generally underpredicted (unless its dimensions are large), and in Figure 2, 
it can be estimated to be somewhere between 0.1 and $0.3 \mathrm{ohm}$, with the late-time estimates being closer to the true value.

\section{Example 2}

In the second example, a $500 \times 500 \mathrm{~m}$ loop is located to the west of the survey grid, the sheet is at a depth of $40 \mathrm{~m}$, and the background

Example 1
$R b=0.5 \mathrm{ohm}$
$R a=0.05 \mathrm{ohm}$
$r=40 \mathrm{~m}$
$z=25 \mathrm{~m}$
$T x=400 \mathrm{~m}$
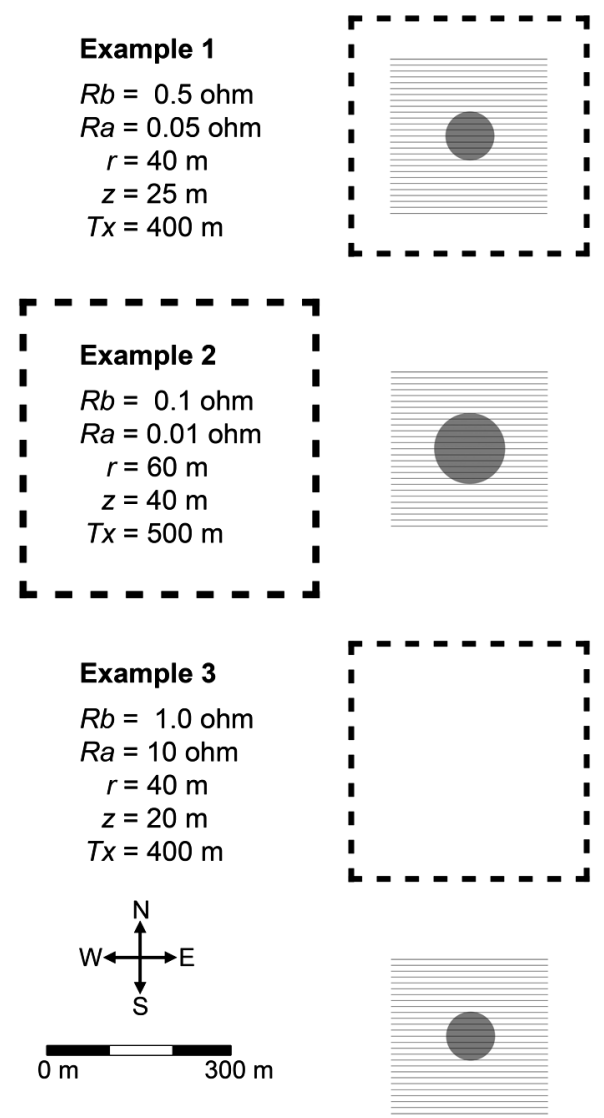

Example 1: Oblique view

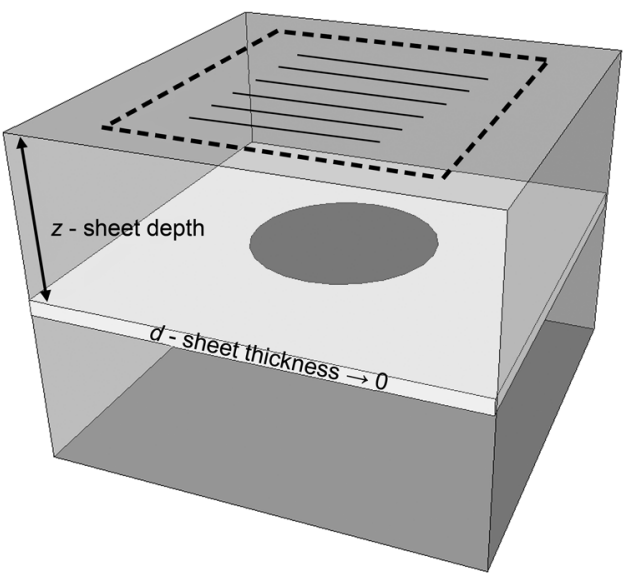

Figure 1. Plan view of the survey geometry used for the three synthetic examples (top, drawn to scale) used in MultiLoop III and, for reference, an oblique view for example 1 (bottom, not drawn to scale, modified from Kolaj and Smith, 2013): Tx represents the dimensions of the square transmitter loop size, and the dark gray circle (of radius, $r$ ) represents the zone of anomalous resistance $(R a)$ within the sheet of background resistance $(R b)$ at a depth of $z$. sheet and anomaly $(r=60 \mathrm{~m})$ resistances are 0.1 and $0.01 \mathrm{ohm}$, respectively (Figure 1). Contrary to example 1, in this example, the secondary horizontal magnetic fields (predominantly $H_{x}^{s}$ ) are comparable in early time channels and larger at intermediate time channels than the secondary vertical magnetic field. The regularization coefficient $\alpha$ was set to zero for the simplified solution and full inversion, and data padding was used for the full inversion. The resistance results gridded along the 27 lines can be seen in Figure 3 .

In this example, both approaches can be used to accurately estimate the background resistance $(0.08$ to $0.1 \mathrm{ohm})$ but the simplified solution produces a conductive and resistive anomaly while the full inversion correctly identifies a single conductive anomaly (slightly offset from the center). If the simplified solution had been used on its own, there may have been some ambiguity as to whether the anomalies are resistive or conductive. Furthermore, uncertainties can also arise if there is a single anomaly on the edge of the grid or on a single line because the interpreter may be unsure if it is truly a single anomaly of the correct resistance or if it is only a portion of a double anomaly (either a conductive or resistive portion).

\section{Example 3}

In the third example, a $400 \times 400 \mathrm{~m}$ loop is located to the north of the survey grid, the sheet is at a depth of $20 \mathrm{~m}$, and the
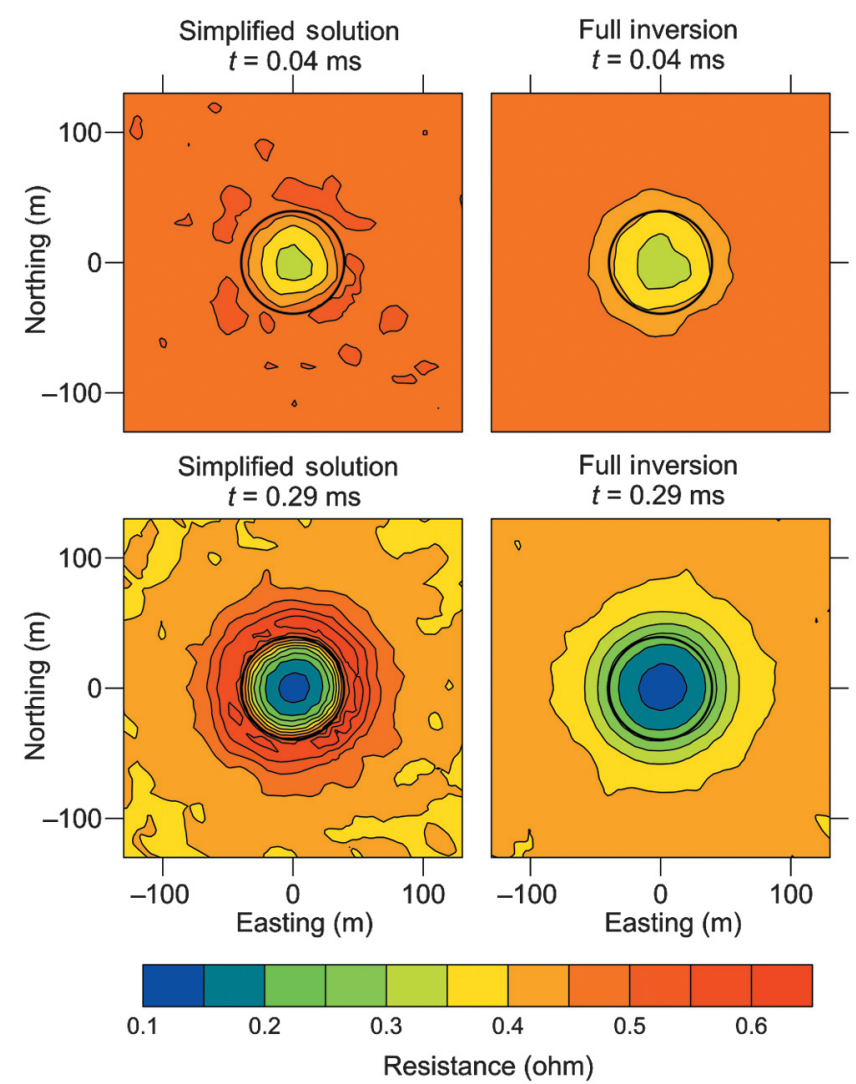

Figure 2. Resistance calculated using the simplified solution (equation 1, left panels) and the full inversion (equations 2 and 4, right panels), at $t=0.04 \mathrm{~ms}$ (top panels) and $t=0.29 \mathrm{~ms}$ (bottom panels) for synthetic example 1 (Figure 1). Resistances of the background sheet and anomaly ( $r=40 \mathrm{~m}$, black circle) are 0.5 and $0.05 \mathrm{ohm}$, respectively. Gridding on this and subsequent figures uses triangulation with linear interpolation. 
background sheet and anomaly $(r=40 \mathrm{~m})$ resistances are 1 and $10 \mathrm{ohm}$, respectively (Figure 1). As with example 2, the secondary horizontal magnetic fields in this survey geometry are comparable to the vertical magnetic field. However, with this model being more resistive and possessing a resistive anomaly (as opposed to a conductive anomaly), the magnetic field strengths are lower than the previous two examples, allowing for numerical noise to be more pronounced (especially in the $d H_{z}^{s} / d z$ ). The calculated resistance using no regularization can be seen in the top two panels of Figure 4. There is a sign reversal in the $d H_{z}^{s} / d z$ at the southern end of the anomaly (away from the loop), and in the simplified solution, this sign reversal causes a negative resistance to be calculated. As such, the absolute value of the resistance with the simplified solution was used. With the full inversion, taking absolute values is unnecessary because the additional terms in the full inversion $\left(H_{y}^{s} d R / d y\right.$ and $H_{x}^{s} d R / d x$ ) that have been ignored in the simplified solution result in positive resistances only. Due to the lower signal value and increased noise, the matrix $\mathbf{A}$ in equation 4 is ill conditioned and, in contrast to the previous two examples, requires regularization in order for a nonerratic solution to be found. A comparison of the two solutions with regularization can be seen in the bottom two panels of Figure 4. For the simplified solution and full inversion, the regularization coefficient $\alpha$ was selected based on an L-curve and qualitative analysis $\left(5 \times 10^{-5}\right.$ and $1 \times 10^{-5}$, respectively). Data padding was used for both methods, and the absolute resistance was used for the simplified solution.

In Figure 4 (bottom panels), the regularized full inversion correctly displays a resistive circular anomaly in the center of the survey grid. The size of the anomaly and background resistance is also consistent with the input model parameters. The regularization helps to remove some of the erratic resistance estimates in the simplified solution. However, overall, the regularization does not aid in its interpretation, and in fact, it reduces the likelihood that it would be correctly interpreted as a resistive anomaly because the conductive portion appears to dominate the map. Moreover, as previously stated, applying regularization to the simplified solution causes the

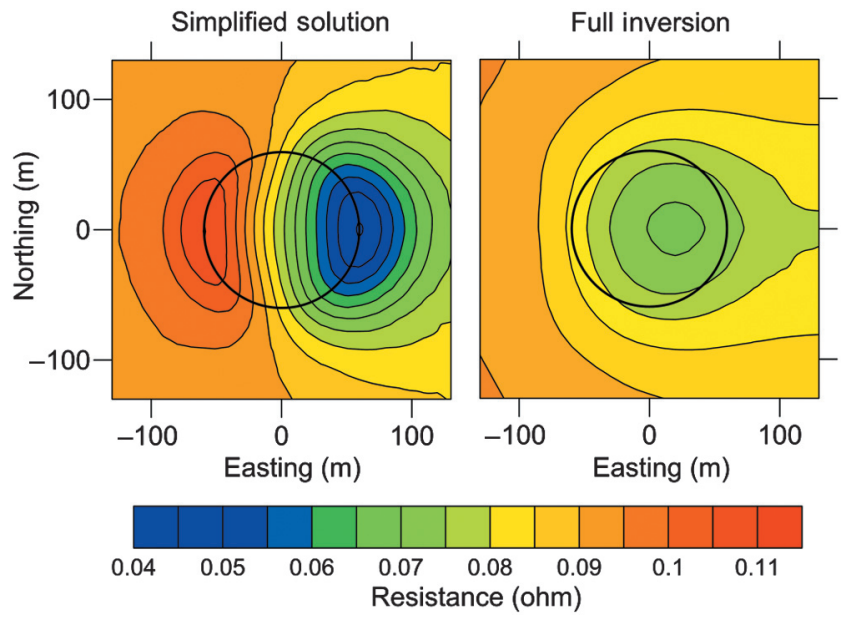

Figure 3. Resistance calculated using the simplified solution (equation 1, left panel) and the full inversion (equations 2 and 4, right panel), at $t=0.04 \mathrm{~ms}$ for synthetic example 2 (Figure 1). Resistances of the background sheet and anomaly $(r=60 \mathrm{~m}$, black circle) are 0.1 and $0.01 \mathrm{ohm}$, respectively. method to lose its advantages over the full inversion. Thus, if regularization is to be applied, then the full inversion is recommended.

\section{Discussion on synthetic examples}

Based on the assumptions of Kolaj and Smith (2013), it is clear that the simplified solution relies on terms 2 and 3 of equation 2 ( $H_{y}^{s} d R / d y$ and $H_{x}^{s} d R / d x$, respectively) being negligible. This occurs when the product of the spatial resistance derivative and horizontal magnetic field component are small in comparison to the product of the resistance and vertical spatial derivative. This can be analytically investigated by calculating an unreliability parameter, the ratio $T$ :

$$
T=100 \frac{\left|\frac{d R}{d y} H_{y}^{s}+\frac{d R}{d x} H_{x}^{s}\right|}{\left|R \frac{d H_{z}^{s}}{d z}\right|},
$$

where $R$ and its spatial derivatives are calculated from the results of the full inversion. A ratio close to zero would imply that terms 2 and 3 are negligible, while a ratio of 100 would imply that the sum of terms 2 and 3 is of the same size as $R d H_{z}^{s} / d z$. The values of $T$ for each example can be seen in Figure 5. Because the resistances, resistance contrasts, and survey geometries differ in each example
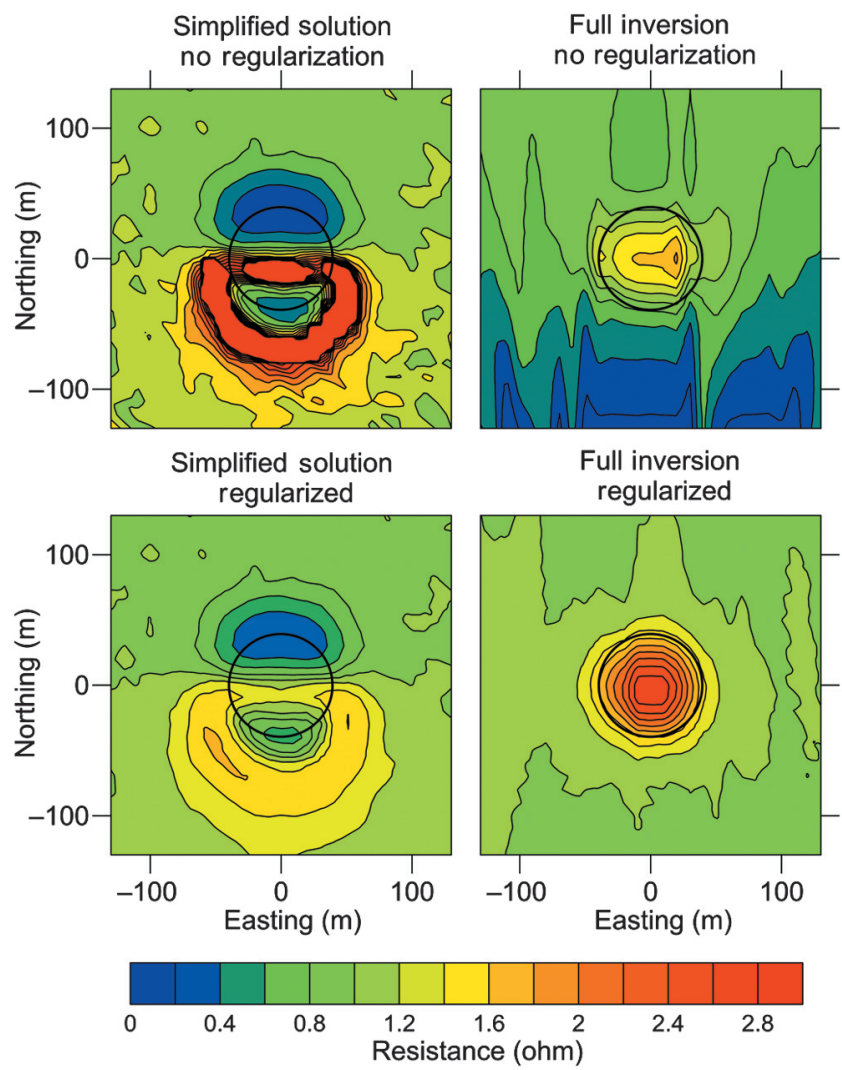

Figure 4. Resistance calculated using and not using regularization (bottom and top panels, respectively) for the simplified solution (equations 1 and 4, left panels) and the full inversion (equations 2 and 4 , right panels) at $t=0.07 \mathrm{~ms}$ for synthetic example 3 (Figure 1). The background sheet and anomaly $(r=40 \mathrm{~m}$, black circle) are 1 and $10 \mathrm{ohm}$, respectively. 
(Figure 1), more attention should be paid to the overall trends (high or low, as opposed to their absolute size) in the $T$ ratio when comparing the three examples in Figure 5.

In example 1, the only area where the simplified solution may yield erroneous resistances is directly adjacent to the conductive anomaly where the ignored spatial resistance derivative is the largest (example 1, Figure 5). This is likely to be what is causing the over-/undershoot on the outside of the anomaly (example 1, Figure 2). Terms 2 and 3 are negligible in the center (i.e., $T \approx 0$ ) where the spatial resistance derivative is small due to the size of the body, which allows for the resistance of the anomaly to be determined more accurately. In examples 2 and 3, the surveys are offset from their transmitter loops and a double anomaly appears in the $T$ ratio maps. Because the relative size of the horizontal magnetic field in comparison to the vertical magnetic field will be higher in this survey geometry (survey offset from the transmitter loop), there is an increased $T$ ratio in examples 2 and 3 . The ratio is not as large further away from the anomaly because the spatial resistance derivatives are much smaller here. As such, the relative contribution of terms 2 and 3 in equation 2 is significant in close proximity to resistance contrasts (anomalies) and by ignoring them, erroneous and misleading results can be generated in the simplified solution (i.e., double anomalies, negative resistances, and over- $/$ undershoots). If the simplified solution is to be used, the survey should be organized in such a way as to minimize the horizontal
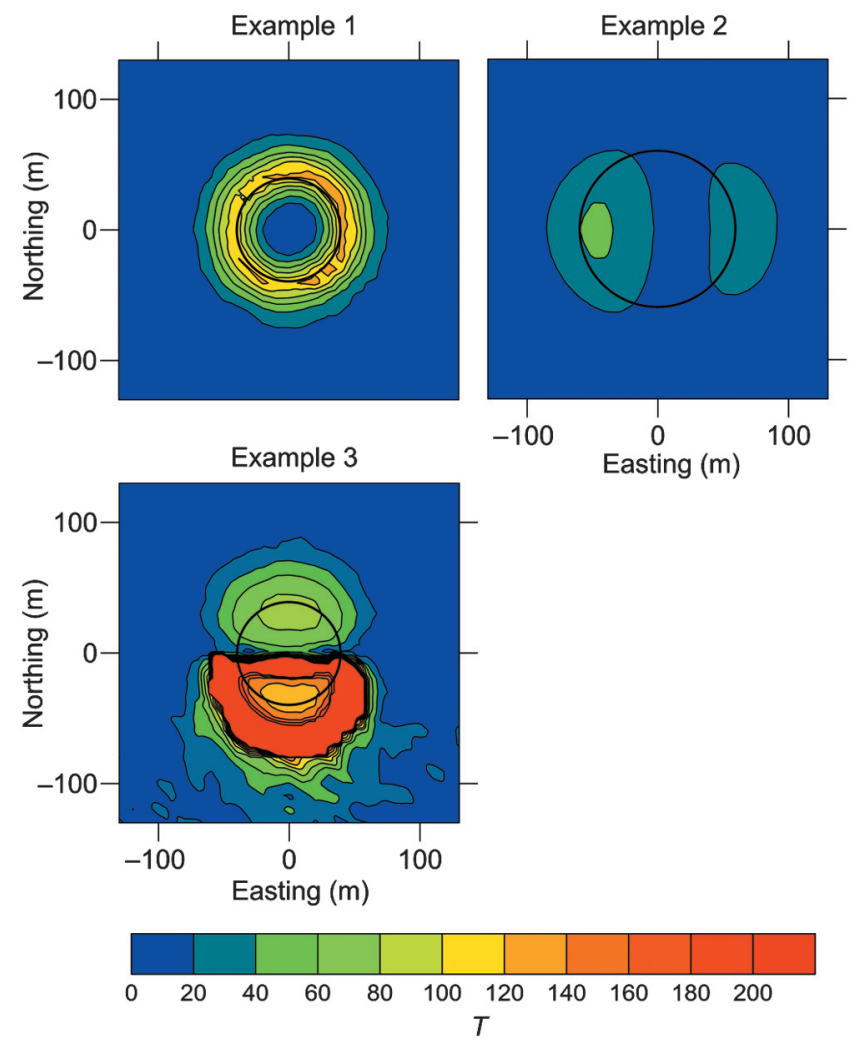

Figure 5. The ratio $T$ (an unreliability parameter), calculated using equation 5 for examples $1(t=0.29 \mathrm{~ms}), 2$, and 3 (regularized example). A high ratio corresponds to areas where terms 2 and 3 of equation 2 are too large to be deemed negligible as they are assumed to be in the simplified solution. Anomaly boundaries are indicated by a black circle. magnetic fields, such as by performing the survey inside of the loop, as is done by Kolaj and Smith (2013). However, even if the survey is performed inside of the transmitter loop, it is still important to investigate the $T$ ratio. In example 1 , if the conductive anomaly had been located closer to one of the transmitter loop edges (example not shown), then a nonsymmetric and potentially large overshoot would have appeared on one side of the anomaly (the side closest to the loop edge where the horizontal magnetic fields are large). Calculating the $T$ ratio would ensure that this overshoot is not incorrectly interpreted as an additional anomaly. Furthermore, caution should also be taken around large resistance contrasts, and the absolute value of the resistance should be used if there are frequent polarity changes in the input variables.

Ideally, we would like to be able to determine where the simplified solution will give erroneous results without having to compute the full inversion. An approximation of the unreliability parameter $T$ is termed $T^{\prime}$, which is identical to equation 5 with the exception that the $R$ and its spatial derivatives from the simplified solution, rather than full inversion, are used. The results of $T^{\prime}$ for each example can be seen in Figure 6. The results are generally more erratic because the simplified solution tends to produce more erratic resistances (i.e., example 3). By comparing Figures 5 and 6, it is revealed that while the $T^{\prime}$ ratio is larger, more erratic, and does not have as well-defined zones as the corresponding $T$ ratio, it is an adequate approximation of $T$. As such, the unreliability parameter $T^{\prime}$ can
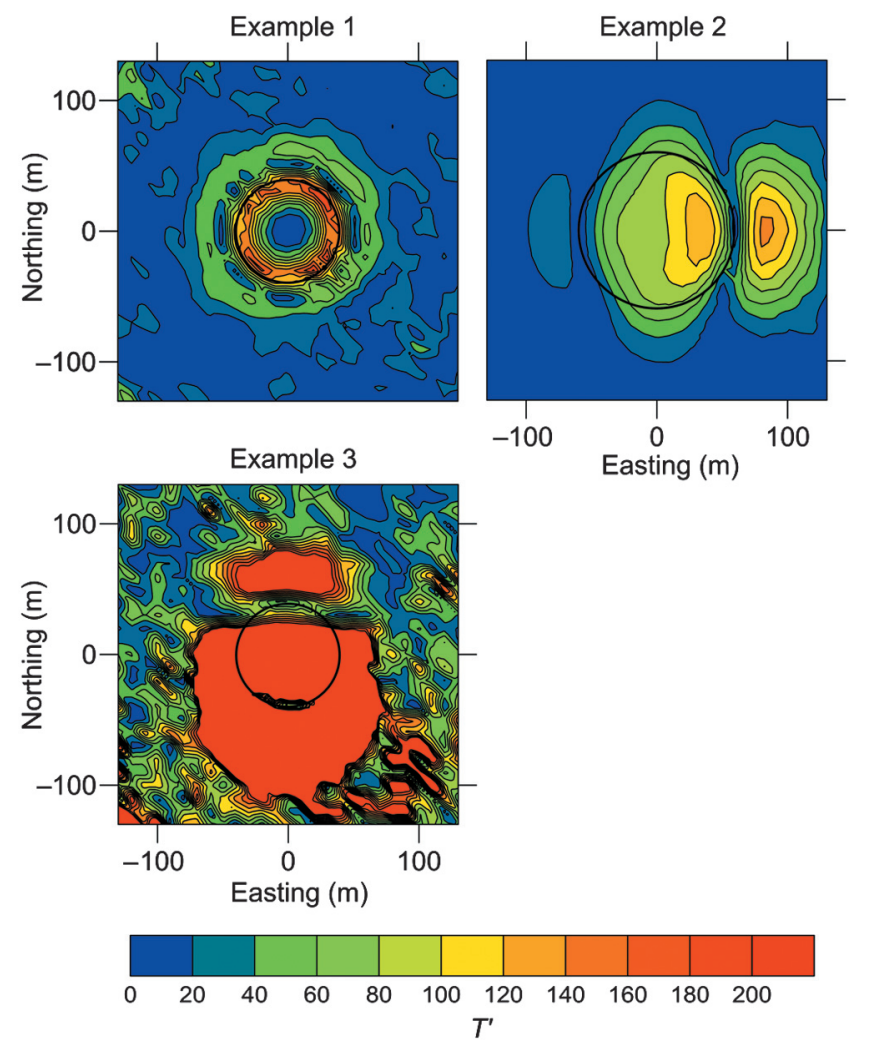

Figure 6. The approximate ratio $T^{\prime}$ (an unreliability parameter), calculated using the resistance and its spatial derivatives from the simplified solution in equation 5 for examples $1 \quad(t=$ $0.29 \mathrm{~ms}$ ), 2, and 3 (nonregularized example). Anomaly boundaries are indicated by a black circle. 
be used to determine where the simplified solution results are unreliable when the full inversion resistance is not available. Unfortunately, both ratios require that the horizontal magnetic field components, which are not required in the simplified solution calculation, are measured.

\section{COMPARISON OF METHODS ON FIELD DATA}

The full inversion is tested on the TDEM survey data of Kolaj and Smith (2013) where the simplified solution had previously been used. A brief description of the survey details are as follows: The survey was performed with the assistance of Vale and Abitibi Geophysics on a dry mine tailings pond situated on Vale property in Sudbury, Ontario, Canada. Three Geonics 3D-3 sensor coils each measuring three components $(x, y$, and $z)$ spaced $1.1 \mathrm{~m}$ vertically apart were used with a SMARTem 24 receiver $(120 \mathrm{kHz}$ sampling rate). A Geonics TEM-57 generated a $30 \mathrm{~Hz} 50 \%$ duty cycle pulse with an exponential turn on and a linear ramp switch off in a loop approximately $700 \times 350 \mathrm{~m}$ (Kolaj and Smith, 2013). The survey was performed inside the loop with a station spacing of $20 \mathrm{~m}$ on five lines spaced $40 \mathrm{~m}$ apart (line length of approximately $200 \mathrm{~m}$, Figure 7). Because the survey was inside the loop, we expected the horizontal components to be small and the simplified solution to work well.

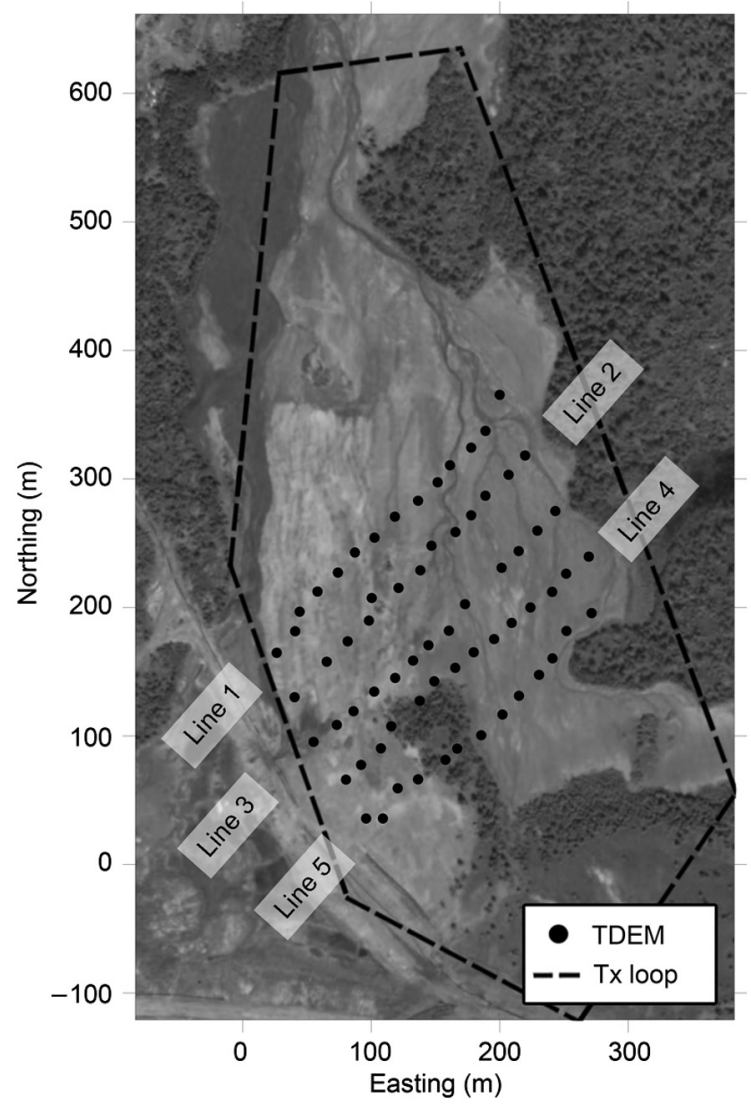

Figure 7. Station locations and loop location for the vertical spatial derivative TDEM survey of Kolaj and Smith (2013) superimposed atop an aerial image of the dry tailings pond on Vale property located in Sudbury, Ontario, Canada (modified from Kolaj and Smith, 2013).
Each station was occupied for 2.5 min (five readings of 756 stacks), and the sensor coil output was integrated to give the magnetic field $\left(B_{x}, B_{y}\right.$, and $\left.B_{z}\right)$ using the full waveform data (Smith and Annan, 2000). Note that the $\mathbf{B}$ rather than $\mathbf{H}$ magnetic fields are used because $\mathbf{B}$ units (nanotesla) are more commonly used in practice ( $\mathbf{B}=\mu_{0} \mathbf{H}$, where $\mu_{0}$ is the magnetic permeability of free space). The $d B_{z} / d z$ was calculated using the difference between the base and the average of the mid and upper sensors (Kolaj and Smith, 2013). Finally, each line was trimmed to only include those stations that were interpreted to be above the thin sheet (Kolaj and Smith, 2013). An example of $d B_{z} / d t, B_{x}, B_{y}$, and $d B_{z} / d z$ for the trimmed line 4 can be seen in Figure 8.

The weighting matrix (error matrix, $\mathbf{W}$ in equation 4 ) for the full inversion was composed of the standard deviation of the five readings of $d B_{z} / d z$ at each station and several stations that were thought to be unreliable due to a poor signal-to-noise ratio ( $\mathrm{S} / \mathrm{N}<3$ for early time and $\mathrm{S} / \mathrm{N}<2$ for intermediate time) were removed entirely (Kolaj and Smith, 2013). Finally, the data were gridded into roughly $5 \times 5 \mathrm{~m}$ cells and the full inversion and simplified solution were
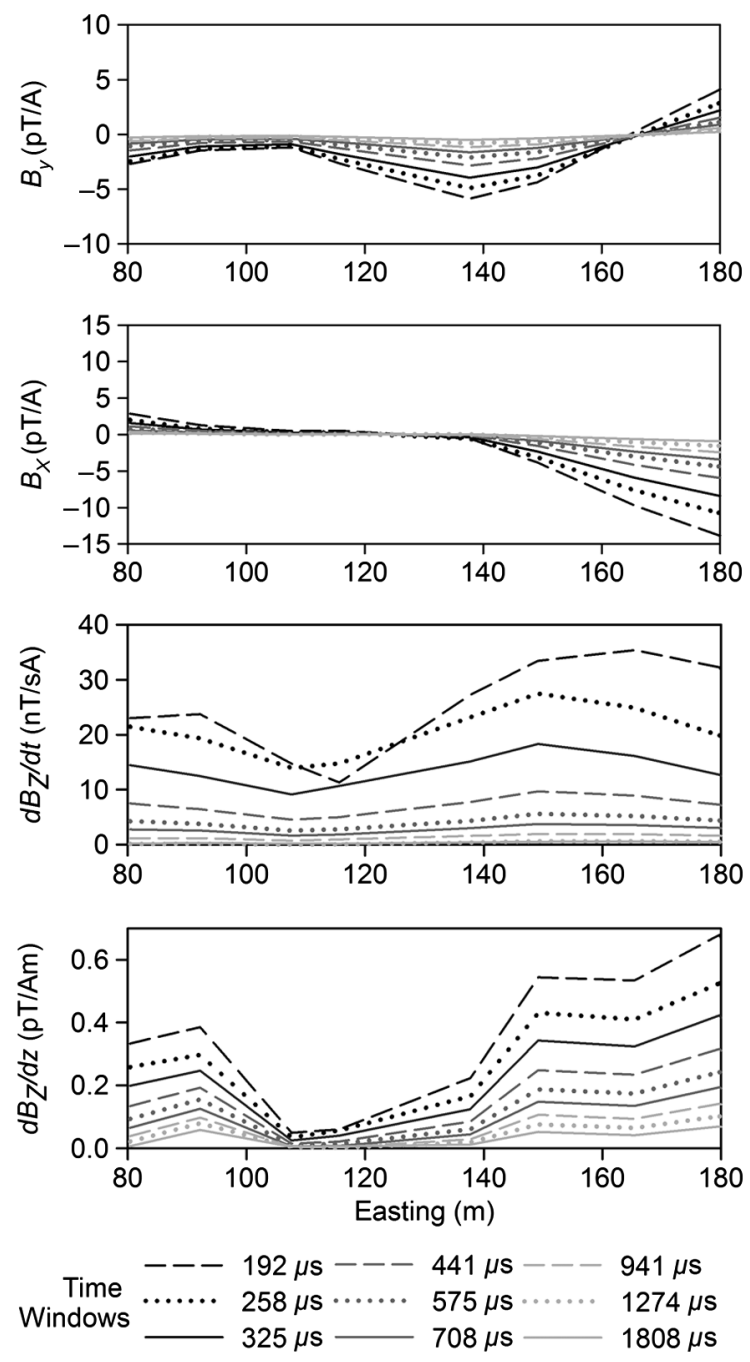

Figure 8. Off-time, windowed, and stacked $B_{y}, B_{x}, d B_{z} / \mathrm{dt}$, and $d B_{z} / d z$ for trimmed line 4 of the TDEM survey of Kolaj and Smith (2013). 
calculated for early and intermediate times (Figure 9). The L-curve analysis suggested that no regularization was required, but it was found that using minor regularization helped to remove some small artifacts in the resistance. As such, the regularization coefficient $\alpha$ was equal to $1.0 \times 10^{-5}$ for the full inversion and no data padding was used. Note that no regularization was used for the simplified solution as suggested above. Apart from the data trimming, the results of the simplified solution (Figure 9) could have been generated in real time in the field. At first glance, the simplified solution and full inversion have the same overall trend of a resistive zone trending roughly from north-south in the middle of the grid. The increase in the calculated conductance from 192 to $575 \mu$ s is consistent with the results found in Kolaj and Smith (2013), where it is attributed to either the finite size of the sheet and/or that the conductivity continues and/or increases with depth.

The differences between the two methods and the validity of the simplified solution can be investigated in more detail in the same manner as in the modeling section (i.e., by calculating the unreliability parameters, the ratio $T$, and/or the approximate ratio $T^{\prime}$ from

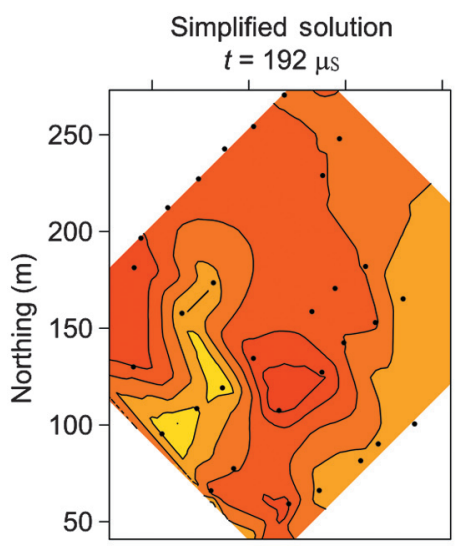

Simplified solution $t=575 \mu \mathrm{s}$

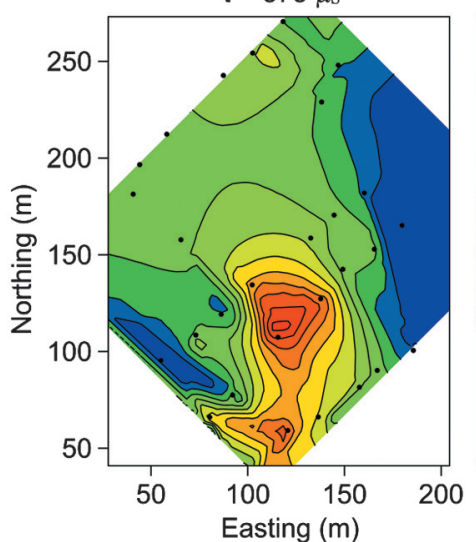

Easting $(m)$

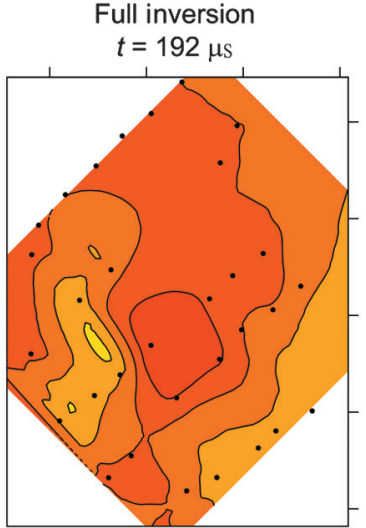

Full inversion $t=575 \mu \mathrm{s}$

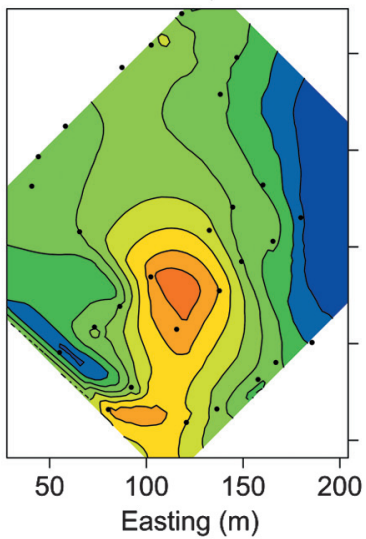

Easting $(\mathrm{m})$

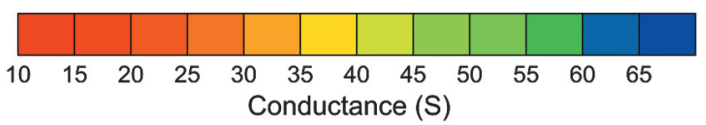

Figure 9. Conductance over the dry tailings pond calculated using the simplified solution (left panels) and the full inversion (right panels) for early time (top panels) and intermediate time (bottom panels). Survey data are from Kolaj and Smith (2013). The black dots represent the station locations with reliable data. equation 5). These results can be seen in Figure 10. Although $T^{\prime}$ is only an approximation, it defines the same general locations as $T$ in Figure 10. The largest ratio occurs overtop the resistive anomalies and somewhat overtop the conductive band seen in the top panels of Figure 9 (not shown well in Figure 10 due to the scale of the color bar). Three profiles have been placed in Figure 10, positioned so that profile 1 is in an area where the ratio $T$ is smallest, while profile 3 has the largest ratio. Based on forward modeling, we do not expect large differences between the simplified solution and the full inversion when the survey is inside the loop. Where there are discrepancies between the methods, it is likely to be an overshoot or undershoot due mainly to lateral changes in resistance (i.e., large values of the resistance derivatives). However, as seen in Figure 8, the horizontal magnetic fields are more significant in certain areas, especially along the eastern end of the survey lines, which may also cause some differences between the conductances calculated with the two methods. In profile 1 , the resistance varies gradually and as such, the resistance gradient is small and there are only minor differences between the two methods and the $T$ and $T^{\prime}$ ratios are small (Figure 11). In profiles 2 and 3, there are more sudden resistance changes, which cause small overshoots and undershoots on the simplified solution results prior to the resistive and conductive anomalies, respectively. For the large resistive anomaly in profile 3,
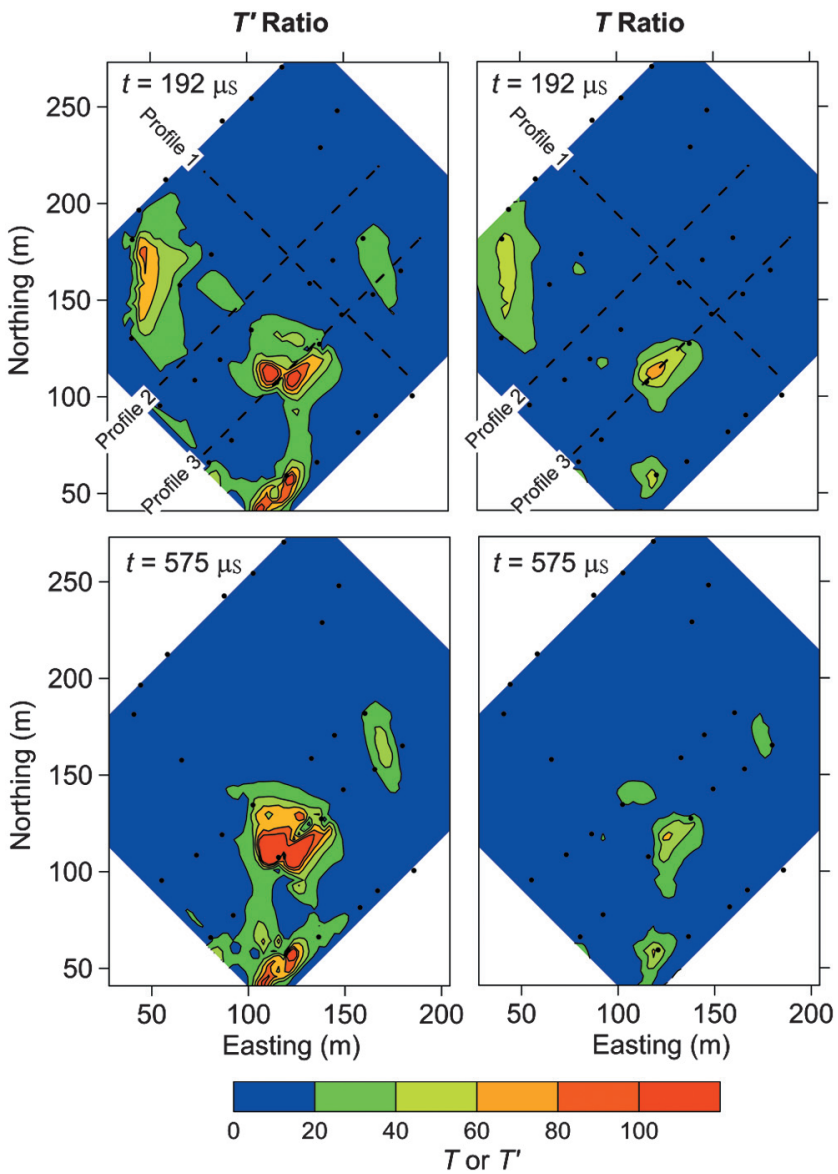

Figure 10. The approximate ratio $T^{\prime}$ and the ratio $T$ (left and right panels, respectively) calculated using equation 5 for Figure 9 at early and intermediate times (top and bottom panels, respectively). The black dots represent the station locations with reliable data. The dashed lines represent the location of the profiles used for Figure 11. 
the full inversion suggests an anomaly that is less resistive than the simplified solution implies. In both profiles, the increased $T$ and $T^{\prime}$ ratio zones coincide well with areas where the conductances differ.

In this field example, although the full inversion does correct some erroneous values found with the simplified solution, the simplified solution does appear to be an adequate approximation. Furthermore, the $T^{\prime}$ ratio (which can be computed without the full inversion and potentially in real time along with the simplified solution) accurately indicated the zones where the simplified solution may be unreliable. Unfortunately, the unreliability parameters ( $T$ or $T^{\prime}$ ) do not indicate in what sense the simplified solution may be erroneous. As such, had only the simplified solution and $T^{\prime}$ ratio been available and had the resistive anomaly been a target of interest, then a follow-up of the full inversion may have been sought. If the simplified solution was done in the field, then the surveyors may have opted to collect more dense data in and around the resistive anomaly to improve the results of the full inversion. If an out-ofloop survey was collected instead, then the synthetic models show that artifacts such as low/high resistance doubles and overshoot and undershoot would have been more prevalent. In this case, it would be more important to look critically at the $T$ or $T^{\prime}$ values.
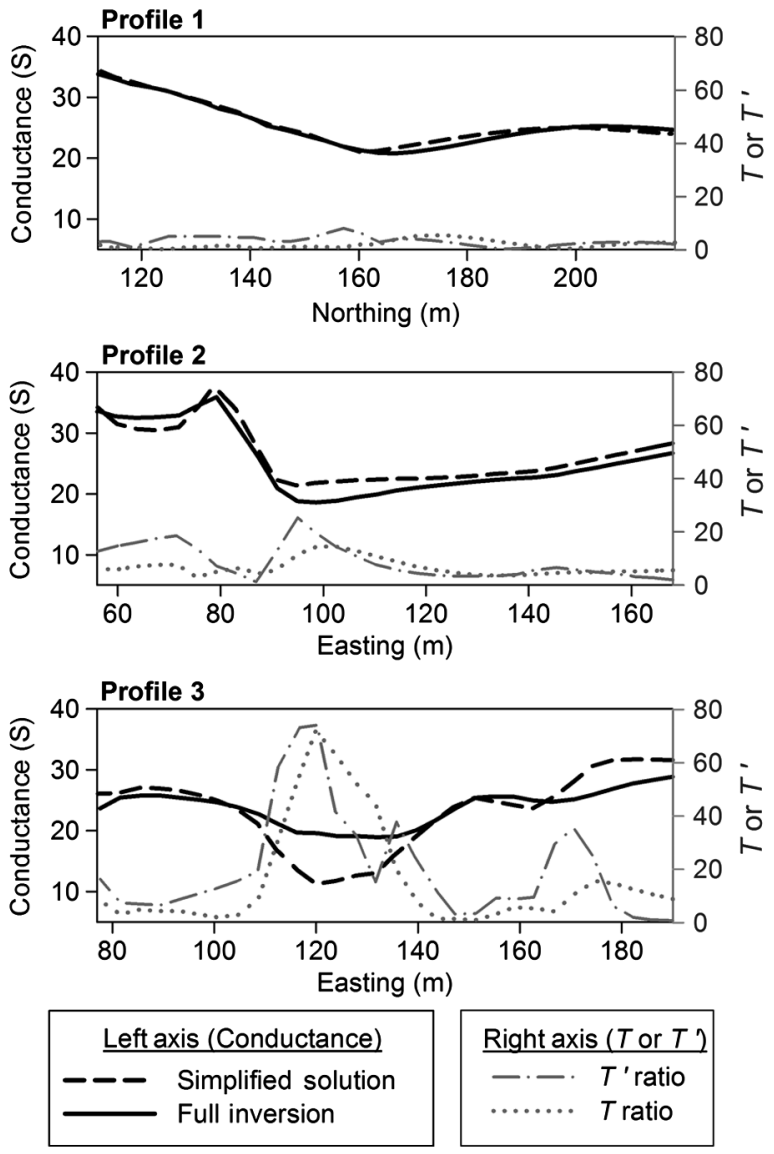

Figure 11. The left axis corresponds to the conductance as calculated using the simplified solution (dashed black line) and full inversion (solid black line) at early time $(t=192 \mu \mathrm{s})$ for the profile locations shown in Figure 10. The right axis corresponds to the corresponding $T^{\prime}$ (dashed-dotted gray line) and $T$ (dotted gray line) ratios.

\section{CONCLUSIONS AND FUTURE WORK}

The differential equation, which describes the EM induction in a laterally extensive thin sheet with a varying resistance, can be solved for resistance using a simplified direct transform of the measured data (the simplified solution) or through inversion (the full inversion). The full-inversion approach is derived for magnetic fields measured directly at the surface of a conductive thin sheet, but, with synthetic models, we showed that reliable resistances can also be calculated above shallow thin sheets. The simplified solution relies on the assumption that $H_{y}^{s} d R / d y$ and $H_{x}^{s} d R / d x$ are negligible in comparison to $R d H_{z}^{s} / d z$, which, we have shown through synthetic modeling, is generally only valid when the survey is performed inside of the loop and where there are minimal resistance contrasts. If the assumptions are invalid, the simplified solution can generate erroneous and misleading results in the form of dipolar (conductive and resistive) anomalies in place of single anomalies. Other erroneous artifacts include negative resistances and estimates that overshoot and/or undershoot near resistance contrasts. By identifying where the ratio $T$ (an unreliability parameter) is large (or the approximate ratio $T^{\prime}$ if the full inversion is not available) the areas where the simplified solution will give erroneous results can be identified. Comparison of the two methods using real field data collected overtop a dry tailings pond in Sudbury, Ontario, Canada, with a fixed in-loop survey geometry showed small differences between the resistances calculated using the two methods, most of which correlated with sudden resistance changes or areas where the horizontal magnetic fields were more significant. The good agreement between the simplified solution and full inversion is consistent with the unreliability parameters $T$ and $T^{\prime}$ being generally less than 100 in the survey area (i.e., the neglected terms in the simplified solution are small in comparison to the terms included).

In the simplified solution, each station is independent of the other and as such, the simplified solution could be performed with a single station. However, the full inversion involves spatial resistance derivatives that are calculated using finite-difference operators and as such, the inversion requires line/grid data (and interpolation if the grid is not adequately dense). The simplified solution also does not require the collection of horizontal magnetic fields, which may save time depending on the equipment used. Furthermore, the simplified solution could be quickly calculated in the field in real time because it only requires simple arithmetic (assuming that the vertical magnetic field and its time derivative are measured or can be calculated from the data) whereas the full inversion is a more complex algorithm that requires some user input (selecting a suitable regularization coefficient and padding the data). As such, the simplified solution is a good candidate as a first pass and/or field interpretation provided that the survey is designed in a way to minimize the horizontal magnetic fields and caution is taken around resistance contrasts. Furthermore, if horizontal magnetic fields are recorded, the ratio $T^{\prime}$ can potentially be calculated alongside the simplified solution in the field (or trivially post data collection), which will indicate zones in which the simplified solution may be providing unreliable conductance estimates. For example, the simplified solution could be performed in the field, possibly with large station spacing, to identify areas of interest and zones where the $T^{\prime}$ ratio is acceptable. If necessary, more finely spaced and/or grid data could then be acquired for the more reliable/accurate full inversion over any areas of interest or zones where the $T^{\prime}$ ratio is found to be 
above some threshold. Whereas the synthetic tests and the field trial focused on fixed-loop TDEM systems, the simplified solution and full inversion can also be used with frequency domain and/or moving (airborne) EM systems.

In this paper, we solved for resistance at each delay time rather than for a single resistance, which is consistent with all delay times. This was done to potentially track for variations in resistance with time that may be associated with changes in the resistivity with depth. In our future work, we aim to use this information to generate resistivity depth sections.

\section{ACKNOWLEDGMENTS}

We are grateful to the following for financial support of this research: NSERC, Vale, Sudbury Integrated Nickel Operations, Wallbridge Mining, KGHM International, and the Centre for Excellence in Mining Innovation. M. Kolaj is grateful for an NSERC Alexander Graham Bell scholarship, an SEG/ARCO scholarship, and a KEGS/ Fugro scholarship. MultiLoop III was provided by Lamontagne Geophysics, and P. Walker guided us in its use. The contour images were generated with Surfer 11 by Golden Software. We also wish to thank Abitibi Geophysics for donating equipment and crew to acquire the field data presented here.

\section{REFERENCES}

Chouteau, M., O. Anterrieu, M. Aubertin, C. Dubreuil-Boisclair, and J. Poisson, 2006, Geophysical characterization of an AMD-generating waste rock pile using ground and borehole techniques: Presented at the 19th EEGS Symposium on the Application of Geophysics to Engineering and Environmental Problems.

Frischknecht, F. C., V. F. Labson, B. R. Spies, and W. L. Anderson, 1991, Profiling methods using small sources, in M. N. Nabighian, ed., Electromagnetic methods in applied geophysics: Applications, Parts A and B: Investigations in Geophysics 2, no. 3, SEG, 105-270.

Grant, F. S., and G. F. West, 1965, Interpretation theory in applied geophysics: McGraw-Hill.

Haber, E., D. W. Oldenburg, and R. Shekhtman, 2007, Inversion of time domain three-dimensional electromagnetic data: Geophysical Journal International, 171, 550-564, doi: 10.1111/j.1365-246X.2007.03365.x.

Hohmann, G. W., and A. P. Raiche, 1987, Inversion of controlled-source electromagnetic data, in M. N. Nabighian, ed., Electromagnetic methods in applied geophysics: Theory: Investigations in Geophysics 1, SEG, 469-503.

Irvine, R. J., and G. Staltari, 1984, Case history illustrating problems in transient electromagnetic surveys: Exploration Geophysics, 15, 155-167, doi: 10.1071/EG984155.

Joshi, M. S., O. P. Gupta, and J. G. Negi, 1988, On the effects of thickness of the half-plane model in HLEM induction prospecting over sulphide dykes in a highly resistive medium: Geophysical Prospecting, 36, 551-558, doi: 10.1111/j.1365-2478.1988.tb02179.x.

Keating, P. B., and D. J. Crossley, 1990, The inversion of time-domain airborne electromagnetic data using the plate model: Geophysics, 55, 705711, doi: 10.1190/1.1442882.

Kolaj, M., and R. S. Smith, 2013, Using spatial derivatives of electromagnetic data to map lateral conductance variations in thin sheet models: applications over mine tailings ponds: Geophysics, 78, no. 5, E225-E235, doi: 10.1190/geo2012-0457.1.
Liu, G., and M. Asten, 1993, Conductance-depth imaging of airborne TEM data: Exploration Geophysics, 24, 655-662, doi: 10.1071/EG993655.

Macnae, J., 2007, Developments in broadband airborne electromagnetics in the past decade, in B. Milkereit, ed., Proceedings of Exploration: Fifth Decennial International Conference on Mineral Exploration: Decennial Mineral Exploration Conferences, 387-398.

Macnae, J. C., and Y. Lamontagne, 1987, Imaging quasi-layered conductive structures by simple processing of transient electromagnetic data: Geophysics, 52, 545-554, doi: 10.1190/1.1442323.

Nabighian, M. N., and J. C. Macnae, 1991, Time domain electromagnetic prospecting methods, in M. N. Nabighian, ed., Electromagnetic methods in applied geophysics: Applications, Part A and B: Investigations in Geophysics 2, SEG, 427-520.

Palacky, G. J., 1987, Resistivity characteristics of geological targets, in M. N. Nabighian, ed., Electromagnetic methods in applied geophysics: Theory: Investigations in Geophysics 1, SEG, 53-125.

Peric, M., 1981, Exploration of Burundi nickeliferous laterites by electrical methods: Geophysical Prospecting, 29, 274-287, doi: 10.1111/j.13652478.1981.tb00405.x.

Price, A. T., 1949, The induction of electric currents in non uniform thin sheets and shells: Quarterly Journal of Mechanics and Applied Mathematics, 2, 283-310, doi: 10.1093/qjmam/2.3.283.

Rutherford, J., T. Munday, J. Meyers, and M. Cooper, 2001, Relationship between regolith materials, petrophysical properties, hydrologeology and mineralisation at the Cawse Ni laterite deposits, Western Australia: Implications for exploring with airborne EM: Exploration Geophysics, 32, 160-170, doi: 10.1071/EG01160.

Sattel, D., and J. C. Macnae, 2001, The feasibility of electromagnetic gradiometer measurements: Geophysical Prospecting, 49, 309-320, doi: 10 .1046/j.1365-2478.2001.00244.x.

Seigel, H. O., and D. H. Pitcher, 1978, Mapping earth conductivities using a multifrequency airborne electromagnetic system: Geophysics, 43, 563575, doi: $10.1190 / 1.1440837$.

Smith, R. S., 2000, The realizable resistive limit: A new concept for mapping geological features spanning a broad range of conductances: Geophysics, 65, 1124-1127, doi: 10.1190/1.1444805.

Smith, R. S., and A. P. Annan, 2000, Using an induction coil sensor to indirectly measure the B-field response in the bandwidth of the transient electromagnetic method: Geophysics, 65, 1489-1494, doi: 10.1190/1 1444837 .

Smith, R. S., and G. F. West, 1987, Electromagnetic induction in an inhomogeneous conductive thin sheet: Geophysics, 52, 1677-1688, doi: 10 $.1190 / 1.1442284$

Swidinsky, A., and R. N. Edwards, 2009, The transient electromagnetic response of a resistive sheet: Straightforward but not trivial: Geophysical Journal International, 179, 1488-1498, doi: 10.1111/j.1365-246X.2009 .04393.x.

Tartaras, E., M. S. Zhdanov, K. Wada, A. Saito, and T. Hara, 2000, Fast imaging of TDEM data based on S-inversion: Journal of Applied Geophysics, 43, 15-32, doi: 10.1016/S0926-9851(99)00030-0.

Walker, P., and Y. Lamontagne, 2006, Electromagnetic interpretation in complex geological environments: 76th Annual International Meeting, SEG, Expanded Abstracts, 1288-1292.

Walker, P., and Y. Lamontagne, 2007, Electromagnetic modeling of the Cree Lake Extension Millenium Deposit, with MultiLoop III, in B. Milkereit, ed., Proceedings of Exploration: Fifth Decennial International Conference on Mineral Exploration, Decennial Mineral Exploration Conferences, $1077-1080$

West, G. F., J. C. Macnae, and Y. Lamontagne, 1984, A time-domain EM system measuring the step response of the ground: Geophysics, 49, 1010 1026, doi: 10.1190/1.1441716.

Zhdanov, M. S., 2002, Geophysical inverse theory and regularization problems: Elsevier Science.

Zhdanov, M. S., 2010, Electromagnetic geophysics: Notes from the past and the road ahead: Geophysics, 75, no. 5, 75A49-75A66, doi: 10.1190/1 .3483901 . 